\title{
Estimation of age dependent penetrance in facioscapulohumeral muscular dystrophy by minimising ascertainment bias
}

\author{
P W LUNT*, D A S COMPSTON \\ From the University of Wales College of Medicine, Heath Park, Cardiff CF4 $4 X W$.
}

SUMMARY In any family study using information gathered retrospectively, the influence of the method of ascertainment on the observed segregation ratio in sibships needs careful consideration. The study of kindred members from outside the area of primary ascertainment is invaluable in providing segregation data with minimal ascertainment bias. For facioscapulohumeral muscular dystrophy (FSHD), using this approach, and based on the presence or absence of characteristic clinical signs rather than on an historical account of age at onset, estimates were derived for penetrance of the FSHD gene of $<5 \%$ for ages 0 to 4 years, $21 \%$ for ages 5 to $9,58 \%$ for ages 10 to $14,86 \%$ for ages 15 to 19 , and $95 \%$ penetrance for age 20 years and over. No difference between families was identified. These figures should facilitate genetic counselling and the interpretation of genetic linkage study results in FSHD.

Facioscapulohumeral muscular dystrophy (FSHD) is inherited as an autosomal dominant disorder; clinical severity and age of onset are very variable. ${ }^{1}$ Although in most cases weakness first affects the facial muscles, causing weakness of eye closure and inability to purse the lips, which can often be recognised in childhood, affected subjects usually present to the clinician in teenage years or early adulthood with symptoms of muscle weakness in one or both shoulder girdles. ${ }^{1}$ A gradual descending progression of weakness to involve muscles of the upper arms, neck, trunk and pelvic girdle, ankle dorsiflexors, and thigh muscles is not infrequent, and can result in requirement for a wheelchair. ${ }^{2}$ There are many mildly affected subjects who are unaware of symptoms, and the presence of recognisable characteristic clinical signs may precede the onset of symptoms in these 'abortive' cases by many years. While the median age of recognisable onset is probably less than 15 years, affected subjects report onset from 3 to 50 years. $^{2}$ The occasional family or isolated case with congenital or infantile onset may represent a separate condition. ${ }^{3}$

In common with the many other dominantly

\footnotetext{
*Present address: Clinical Genetics Unit. Institute of Child Health. Bristol Children's Hospital, Bristol BS2 8BJ.

tPresent address: Department of Neurology. University of Cambridge. Addenbrooke's Hospital, Cambridge.

Received for publication 8 June 1988.

Revised version accepted for publication 28 June 1989.
}

inherited conditions that show similar variability in the severity of expression and in the age at onset, knowledge of the age dependent penetrance is required for estimating the risk of 'carrier' status for those who are normal on examination, the likely severity in affected offspring, and the weighting appropriate for apparently unaffected subjects in genetic linkage studies. Previous estimates of penetrance for the FSHD gene range from 82 to $94 \%$ in 'younger' age groups ${ }^{2}{ }^{4}$ and from 92 to $100 \%$ in 'older' age groups. ${ }^{24-6}$ From a review of these, and other previous surveys, it is clear that reliable values are still required, as the retrospective nature of the ascertainment may have introduced significant bias.

As a prospective cohort study is not, however, a practicable proposition, age dependent penetrance can only be derived from data collected retrospectively. Two simple methods for estimation of penetrance, applicable to dominantly inherited conditions, are as follows. ${ }^{7}$

(1) Weinberg's abridged census method: a crosssectional study of the age dependent ratio of affected:unaffected at risk subjects.

(2) Actuarial method: an actuarial life table based on current age if unaffected, and subjective assessment of age at onset if affected.

Because of the frequency of 'abortive' cases in FSHD, use of an actuarial life table based on anamnestic data for onset of symptoms is inappro755 
normal on examination. For estimation of age dependent penetrance, although a cross sectional analysis would be invalid if lifespan were reduced, ${ }^{7}$ this is rarely the case in FSHD ${ }^{89}$ and enables Weinberg's abridged census method to be the method of choice. In any dominantly inherited condition, segregation analysis should include only those who have the same prior probability of heterozygote status following retrospective ascertainment of their at risk situation. Limitation of analysis to offspring of previously proven affected subjects would provide insufficient information about older age groups. Hence, particular care in analysis is required for those subjects whose at risk situation is defined only by the recognition that they themselves, a sib, or offspring are affected.

We present data for age dependent penetrance derived from a survey of FSHD families, in which bias of analysis has been minimised by critical review of ascertainment. Comparison is also made between reported ages at onset in parents and offspring to test for any significant familial correlation or influence of sex of affected parent.

\section{Methods and examples}

All subjects with a diagnosis of FSHD supported by EMG or muscle biopsy or both, and previously known to the Departments of Genetics or Neurology at the University Hospital of Wales, were contacted. Home visits were made to as many family members as possible, and clinical status scored after examination and serum creatine kinase (CK) assay. Affected subjects were also asked to estimate their age at onset of the disease. Weakness in any muscle group was assessed on the MRC scale. ${ }^{10}$ This grading was used in interpretation of the significance of minimally abnormal clinical signs in otherwise asymptomatic persons, through definition of empirical criteria for assigning affected status (table 1).

As some families from outside the primary study area (that is, from outside Wales) had been referred specifically to assist with genetic linkage studies, the following inclusive criteria were used to identify 145 subjects from 15 families for estimation of penetrance: (1) proband resident in Wales; (2) proven dominant inheritance; (3) subjects who had been examined by one of us (PWL). In addition one branch of a previously reported large family from outside Wales ${ }^{11}$ was included where there was no previous knowledge of the status of members of that particular branch.

Patients were categorised by their relationship to the proband and route of ascertainment of at risk status (table 2). Sibs of probands and of their
TABLE 1 FSHD: clinical criteria for affected status.

Affected status requires:

Family history plus: 2 hard signs or 1 hard and 2 soft signs or 4 soft signs

Hard signs Obvious wasting and/or strength MRC 4- or weaker in a characteristic muscle group*; each muscle group involved to be counted as a separate sign.

Serum CK $>1.5 \times$ upper limit of normal.

Soft signs Muscle strength MRC 4 or $4+$ in a characteristic group*; each muscle group involved to be counted as a separate sign. Serum CK: 1-1.5 xupper limit of normal. Aching shoulders on history.

History of sleeping with eyes open.

Characteristic muscle groups affected

Orbicularis oculi (eye closure)

Perioral muscles (cheek puff, pout, whistle)

Shoulder girdle muscles (including scapular winging)

Triceps or biceps brachii

Tibialis anterior, peroneal group (ankle dorsiflexion)

Proximal lower limb muscles.

TABLE 2 Ascertainment categories and inclusion in segregation analysis.

\begin{tabular}{|c|c|c|c|}
\hline Category & Ascertainment & $\begin{array}{l}\text { Inclusion in } \\
\text { segregation } \\
\text { analysis }\end{array}$ & $\begin{array}{l}\text { Entries } \\
\text { required }\end{array}$ \\
\hline $\mathbf{A}$ & Probands & - & - \\
\hline B & $\begin{array}{l}\text { Affected parent or grandparent } \\
\text { of propands }\end{array}$ & - & 음 \\
\hline C & $\begin{array}{l}\text { Offspring of proband or of previously } \\
\text { known affected relative }\end{array}$ & + & Single \\
\hline D & $\begin{array}{l}\text { Sibs of probands or of affected parent, } \\
\text { resident in Wales, and with parent } \\
\text { or grandparent (respectively) } \\
\text { independently known to be affected }\end{array}$ & - & - \\
\hline $\mathbf{E}$ & As for $\mathrm{D}$ but resident outside Wales & + & Single \\
\hline $\mathbf{F}$ & $\begin{array}{l}\text { Sibs of an affected subject, whose } \\
\text { affected status proves parent to be } \\
\text { an obligate heterozygote, or proves } \\
\text { dominant inheritance }\end{array}$ & + & Multiple \\
\hline G & Living 'obligate' heterozygotes & + & Double \\
\hline $\mathbf{H}$ & Living ancestors at obligate $50 \%$ risk & + & Single \\
\hline $\mathbf{Q}$ & Others/unscorable/dead & - & - \\
\hline
\end{tabular}

parents were also categorised by residence within or outside the primary ascertainment area (Wales). The prior risk of heterozygote status was assessed for each category and those persons sharing a 50\% risk were used for estimation of the age dependent penetrance calculated from the observed segregation ratios for each age group as: [ $2 \times$ number of affected/ number of (affected+unaffected)]. In some categories a $50 \%$ prior risk applies to the subjects included only if the sibships from which they come are repeatedly entered for each additional affected member, equivalent to methods for complete truncate ascertainment. ${ }^{12}$ This applies to all offspring of an affected subject who is proven to be a heterozygote only through recognition of affected status in any one of the offspring. In this situation the obligate heterozygote parent, if examined, was also 
entered twice to accommodate the $100 \%$ risk of heterozygote status.

EXAMPLE 1: FAMILY FSH 028 (FIG 1a)

Although III.1 was said to have had muscular dystrophy, her records could not be traced and dominant inheritance was proven by examination of III.5, whose mother (II.2) was not previously known to have been affected. Since the probability of II. 2 having one living affected offspring is twice that of both offspring being affected, the prior risk of heterozygote status for the other offspring (III.4) would be $1 / 3$ rather than $1 / 2$. However, III. 4 can be included (category $F$ ) provided that, if in the equivalent situation with both offspring affected, they would be entered once each, resulting in an equal segregation ratio when averaged over all such families. The 42 year old sister (IV.1) of the proband was clinically unaffected. Two of her daughters satisifed the diagnostic criteria given in table 1 and were classed as minimally affected, thereby proving IV.1 to be an obligate carrier. Each of the affected daughters was considered separately, such that V.1 and V.2 were listed once each in category $F$ and their unaffected sister (V.3) was included twice. IV.1 is listed twice in category G.

EXAMPLE 2: FAMILY FSH 004 (FIG 1b)

As neither of the parents (I.1, I.2) of the proband (II.1) was known to have been affected, the proof of dominant inheritance in this family followed the

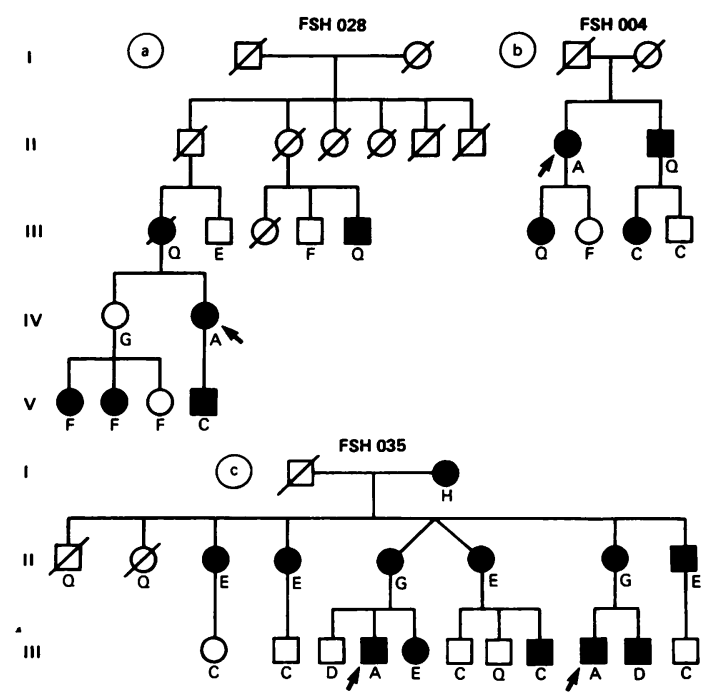

FIG 1 Three pedigrees selected to illustrate classification of ascertainment categories. (a) FSH 028, (b) FSH 004, (c) FSH 035. diagnosis of FSHD in one of the daughters (III.1) of the index case. There will be twice as many such families with one daughter affected as with both affected and hence the prior risk of heterozygote status for the other daughter would be $1 / 3$ rather than $1 / 2$. She can also be included in category $F$ as the situation for her sibship is equivalent to that of complete truncate ascertainment. II. 2 cannot be scored, as only by his affected status could it be proven that his sister (II.1) did not represent a new mutation. His offspring was seen without any prior knowledge of their status and were included in category $\mathrm{C}$.

In multiply ascertained sibships, sibs from outside the study area were included once only.

EXAMPLE 3：FAMILY FSH 035 (FIG 1c)

This family was ascertained independently through both III.4 and III.9. Their mothers (II.5 and II.7) were obligate heterozygotes (category $G$ ) and their grandmother (I.2) had a true $50 \%$ risk of heterozygote status before examination (category $\mathrm{H}$ ). Welsh sibs of the probands (category D) were excluded from analysis as their prior risk of heterozygote status would be less than $50 \%$ unless the chance of ascertainment of a sibship was directly proportional to the number of affected sibs. ${ }^{13}$ Sibs (II.3, II.4, II.6, II.8) of their parents, living outside Wales, have no chance of primary ascertainment and were entered once each in category $E$ together

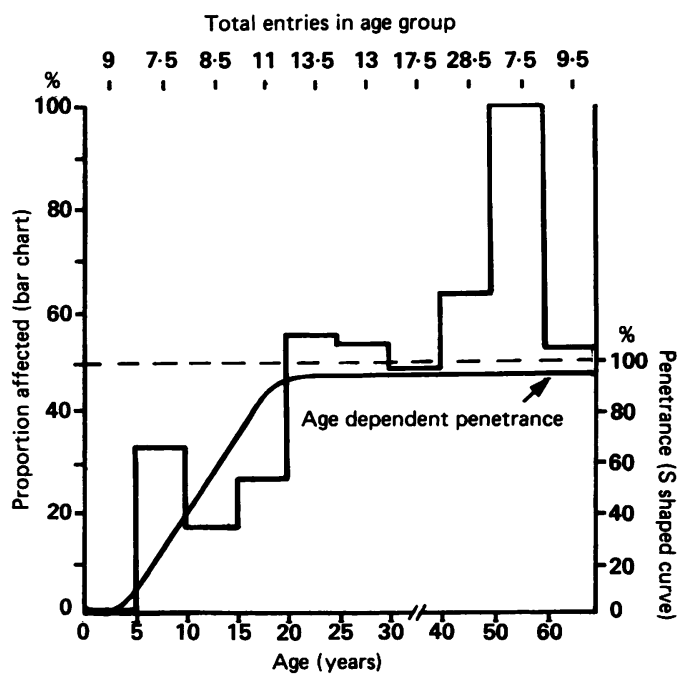

FIG 2 Age dependent penetrance of FSHD (S shaped curve) extrapolated from proportion clinically affected among all entries at $50 \%$ prior risk of heterozygote status (bar chart). Penetrance $=2 \times$ proportion affected. Half entries result from half weighting of one family. 
with non-Welsh sibs of probands for whom the same situation applies.

The Welsh proband of one large family was identified and referred after an extended family study by another centre. ${ }^{14}$ As large families with a high proportion of affected members are the ones most likely to have been studied previously, in order to minimise the bias that would result from either exclusion or full inclusion of this family, all members were empirically given half weight and classed as half subjects in fig 2 .

\section{Results}

Penetrance values (table 3 ) have been extrapolated graphically from the number and proportion of clinically affected or unaffected entries at $50 \%$ prior risk of heterozygote status in each age group (fig 2). The results suggest penetrance of the FSHD gene of $<5 \%$ for ages 0 to 4 years; $21 \%$ for ages 5 to $9 ; 58 \%$ for ages 10 to $14 ; 86 \%$ for ages 15 to 19 ; and $95 \%$ penetrance over age 20 . The median age at onset (age for $50 \%$ penetrance) is 11.5 years. Conversely, persons born at $50 \%$ risk of FSHD heterozygote status still carry a $12 \%$ risk if they are asymptomatic but in the age group 15 to 19 years, and $5 \%$ risk if aged 20 years or over.

For all families ascertained through probands in Wales, there was no correlation between the reported age at onset (or age at first recognition of clinical signs) of affected offspring with that of their affected

TABLE 3 Penetrance and risk to asymptomatics.

\begin{tabular}{|c|c|c|c|c|c|}
\hline & \multicolumn{5}{|c|}{ Age group (y) } \\
\hline & $0-4$ & $5-9$ & $10-14$ & $15-19$ & $20+$ \\
\hline $\begin{array}{l}\text { Penetrance }=\text { proportion of } \\
\text { FSHD heterozygotes } \\
\text { clinically detectable }(x) \\
\text { Proportion of asymptomatics } \\
\text { at } 50 \% \text { congenital risk who } \\
\text { will be FSHD heterozygotes } \\
(1-x / 2-x)\end{array}$ & $\begin{array}{l}<5 \% \\
50 \% \\
50\end{array}$ & $21 \%$ & $58 \%$ & $86 \%$ & $95 \%$ \\
\hline
\end{tabular}

TABLE 4 Reported age at onset in offspring by sex and reported age at onset in affected parent.

\begin{tabular}{|c|c|c|c|c|c|c|}
\hline \multirow{2}{*}{\multicolumn{2}{|c|}{$\begin{array}{l}\text { Sex and age at } \\
\text { onset in offspring (y) }\end{array}$}} & \multicolumn{5}{|c|}{ Sex and age at onset of affected parent (y) } \\
\hline & & \multicolumn{2}{|l|}{ Male } & \multicolumn{2}{|c|}{ Female } & \multirow[t]{2}{*}{ Total } \\
\hline & & $0-19$ & $20+$ & $0-19$ & $20+$ & \\
\hline \multirow[t]{2}{*}{ Male } & $0-19$ & 6 & 5 & 14 & 13 & 38 \\
\hline & $20+$ & 1 & 4 & 2 & 5 & 12 \\
\hline \multirow[t]{2}{*}{ Female } & $0-19$ & 5 & 5 & 10 & 8 & 28 \\
\hline & $20+$ & 3 & 2 & 3 & 8 & 16 \\
\hline \multicolumn{2}{|c|}{ Total } & 15 & 16 & 29 & 34 & 94 \\
\hline
\end{tabular}

$\chi^{2}(9 \mathrm{df})=6 \cdot 7 ; p>0 \cdot 5$.
TABLE 5 Reported age at onset among affected members of the six largest families.

\begin{tabular}{|c|c|c|c|c|c|}
\hline \multirow[t]{2}{*}{ Family } & \multirow{2}{*}{$\begin{array}{l}\text { Total } \\
\text { affected }\end{array}$} & \multicolumn{4}{|c|}{ Reported age at onset $(y)$} \\
\hline & & $0-9$ & $10-19$ & $20-29$ & $30+$ \\
\hline FSH 001 & 17 & 3 & 6 & 4 & 4 \\
\hline FSH 067 & 17 & 2 & 8 & 2 & 5 \\
\hline FSH 009 & 15 & 1 & 10 & 3 & 1 \\
\hline FSH 006 & 12 & 1 & 2 & 4 & 5 \\
\hline FSH 035 & 12 & 0 & 5 & 5 & 2 \\
\hline FSH 025 & 10 & 1 & 3 & 4 & $\overline{2}$ \\
\hline Total & 83 & 8 & 34 & 22 & 19 \\
\hline
\end{tabular}

parent, or with their sex (table 4). There was no evidence for heterogeneity in the distribution of reported age at onset (or age at first recognition of clinical signs) between those six families that had at least 10 affected subjects (table 5). Age at onset does not therefore appear to be familial or related to sex.

\section{Discussion}

Differential diagnosis within a dominantly inherited 'facioscapulohumeral syndrome' includes spinal muscular atrophy, mitochondrial myopathy, and muscular dystrophy. ${ }^{8}$ Clinical and pathological dis tinction between these diagnoses is difficult, and theo recent finding of dystrophin gene deletions typical of Becker muscular dystrophy in males previously diagnosed as having $X$ linked spinal muscular atrophy ${ }^{15}$ lends support to views that dominantly inherited FSH syndrome may be a single genetic condition. ${ }^{2}$ We considered all the families included to have a single genetic entity, with EMG or biopsy findings supportive of a diagnosis of muscular dystrophy, and for the estimation of penetrance have therefore combined the data from our several families.

In estimation of age dependent penetrance in dominantly inherited conditions from retrospective analysis of segregation data, offspring of affected subjects will not all share the same probability of affected status, and only those with a $50 \%$ prior probability can be included. This is of particular importance in three situations: (1) where a parent is proven to be affected only through recognition of affected offspring; (2) where dominant inheritance can only be proven through there being an affected member of the sibship under study; and (3) for sibs of the proband or of the affected parent.

Situations 1 and 2 are both equivalent to complete truncate ascertainment of the sibships studied, and should be analysed accordingly by considering each affected offspring as a separate proband and rescoring the segregation ratio among the sibs of each in 
turn. ${ }^{12}$ In situation 3 , sibs from outside the study area will each be at $50 \%$ risk of heterozygote status. Sibs living within the study area will only be at $50 \%$ prior risk, and hence Weinberg's proband method ${ }^{12}$ will only be applicable if the chance of primary ascertainment of the sibship is sufficiently low to approximate to proportionality with the number of affected members of the sibships living in the study area. ${ }^{13}$ In this study, in which no attempt was made to identify every case in Wales, there were 30 probands from a population of 2.8 million. Using the highest previous prevalence figure of $1 / 20000,{ }^{4}$ we anticipate a total of 140 cases of FSHD in Wales, and hence estimate that the chance of primary ascertainment as a proband for this study was $21 \%$ (30/140), too high for Weinberg's proband method to be valid. The decision to exclude Welsh sibs of probands from analysis is supported by the observation of a segregation ratio among members of this group aged 20 years or over of $0 \cdot 28$ (10 affected:23 unaffected), confirming the expectation of a segregation ratio significantly less than $50 \%$.

Previous studies may both overestimate and underestimate penetrance for the FSHD gene. Penetrance values based on the reported age of onset of symptoms, such as Chung and Morton's value $^{6}$ of $87 \%$ penetrance by the age of 20 , underestimate the proportion with recognisable clinical signs. Becker's estimates of penetrance 4 of $82 \%$ for sibs of probands, and $92 \%$ for sibs of their parents, are also likely to be underestimates, as analysis was by Weinberg's proband method in a study that gave a high prevalence for FSHD and therefore in which a high level of primary ascertainment can be assumed. Overestimation of penetrance owing to inclusion of sibships where the affected status of the parent is confirmed only through identifying an affected offspring is likely in studies of a single extended family, such as that of Tyler and Stephens, ${ }^{5}$ which suggested a $92.2 \%$ penetrance across all ages. Padberg's results ${ }^{2}$ of $89.7 \%$ at the age of $10,94.3 \%$ at 20 years, and $99 \%$ at 30 years may be the most reliable figures available until now, as overestimation of penetrance in the larger families may be offset by underestimation from the sibships of probands in the smaller families.

We found a slight excess of affected subjects in the over 20 age groups (53.5 affected:36 unaffected), but this did not differ significantly from a 1:1 ratio. Although our results suggest full penetrance by the age of 20 , an upper limit of $95 \%$ penetrance has been chosen because in the wider gene mapping study there were 45 obligate carriers, of whom two (aged 42 and 51) were clinically unaffected. Our results are in broad agreement with previous publications, but suggest lower penetrance values for children. This may reflect both a greater emphasis on the necessity for independence of status and ascertainment in considering the offspring of obligate carriers, and a stricter definition of affected status (table 1) than has been used previously. Several subjects for whom clinical status was initially in doubt, but who had been classed as affected in a previous clinical study, ${ }^{16}$ were scored as unaffected by our criteria.

We found no correlation between the age at onset of offspring with that reported by their parents, no difference according to sex or severity of affected parent, and no tendency for sibs to have a similar age of onset, except as could be accounted for by similarity in age at first examination. There was no significant difference between the distribution of ages of onset in the six largest families. Consequently we feel that these penetrance figures can be applied to all families showing a dominantly inherited FSH syndrome, and are independent of the sex or severity of the affected parent. These figures should facilitate genetic counselling and the interpretation of genetic linkage study results in FSHD.

We wish to thank the members of the various families for their help and cooperation in this study, and the various clinicians, in particular Dr Robin Fitzsimons, for introduction to several of these families. PWL was funded by the Muscular Dystrophy Group of Great Britain.

\section{References}

1 Dubowitz V. Muscle disorders in childhood. Philadelphia: Saunders, 1978.

2 Padberg G. Facioscapulohumeral disease. MD thesis, University of Leiden, 1982.

3 Bailey RO, Marzulo DC, Hans MB. Infantile facioscapulohumeral muscular dystrophy: ncw observations. Acta Neurol Scand 1986;74:51-8.

4 Becker PE. Dystrophia musculorum progressiva. Eine genetische und klinische untersuchung der muskeldystrophien. Stuttgart: Georg Thieme Verlag, 1953.

5 Tyler FH, Stevens FE. Studies in disorders of muscle. II. Clinical manifestations and inheritance of facioscapulohumeral dystrophy in a large family. Ann Intern Med 1950;32:640-60.

6 Chung CS, Morton NE. Discrimination of genetic entities in muscular dystrophy. Am J Hum Genet 1959;11:339-59.

7 Chase GA, Folstein MF, Breitner JCS, et al. The use of life tables and survival analysis in testing genetic hypotheses, with application to Alzheimer's disease. Am J Epidemiol 1983;117: 590-7.

8 Gardner-Medwin D. Clinical features and classification of the muscular dystrophies. Br Med Bull 1980;36:109-15.

9 Prot J. Genetic-epidemiological studies in progressive muscular dystrophy. J Med Genet 1971;8:90-5.

10 Medical Research Council. Aids to the examination of the peripheral nervous system. Memorandum no 45. London: HMSO, 1982.

11 Boyes JW, Fraser FC, Lawler SD, et al. A pedigree of progressive muscular dystrophy. Ann Eugen 1950;15:46-51.

12 Stern C. Principles of human genetics. 3rd ed, chap 10. San Francisco: Freeman, 1973. 
13 Morton NE. Outline of genetic epidemiology. Chap 4 and 5. Basel: Karger, 1982.

14 Edwards RHT, Griffiths RD, Hayward M, et al. Modern methods of diagnosis of muscle diseases. $J R$ Coll Physicians Lond 1986;20:49-55.

${ }^{15}$ Lunt PW, Cumming WJK, Kingston $\mathrm{H}$, et al. DNA probes in differential diagnosis of Becker muscular dystrophy and spinal muscular atrophy. Lancet 1989;i:46-7.
${ }^{16}$ Fitzsimons RB, Gurwin EB, Bird AC. Retinal vascular abnormalities in facioscapulohumeral muscular dystrophy. Brain 1987;110:631-48.

Correspondence to Dr P W Lunt, Clinical Genetics Unit, Institute of Child Health, Bristol Children's Hospital, St Michael's Hill, Bristol BS2 8BJ. 